\title{
Bridging to transplantation with left ventricular assist devices: Outcomes in patients aged 60 years and older
}

Veli K. Topkara, MD, Nicholas C. Dang, MD, Timothy P. Martens, MD, Faisal H. Cheema, MD, Judy F. Liu, BA, Michael Argenziano, MD, and Yoshifumi Naka, MD, PhD, New York, NY

$\mathrm{W}$ ith the rapid growth in the elderly segment of the population, an increasing number of older patients will have end-stage heart failure and require cardiac surgery. ${ }^{1}$ The demographic of patients undergoing left ventricular assist device (LVAD) implantation is expected to shift toward older and sicker patients in parallel. This study was designed to determine the effect of advanced age on postimplantation complications, bridge-to-transplantation rates, and posttransplantation survival in LVAD recipients.

\section{Patients and Methods}

Two hundred one patients undergoing LVAD implantation at a single center from July 1996 through April 2004 were retrospectively reviewed. Patients were categorized according to 3 age cohorts: group 1, less than 40 years; group 2, 40 to 59 years; and group 3, 60 years or older. These groups were then compared with regard to baseline characteristics, LVAD scores, ${ }^{2}$ postoperative complications, bridge-to-transplantation rates, and posttransplantation survival.

Data were represented as frequency distributions and percentages. Values of continuous variables were expressed as means \pm SD. Continuous variables were compared by the analysis of variance test, whereas nominal variables were compared using $\chi^{2}$ tests. Kaplan-Meier analysis was used to calculate long-term survival along with $\log$-rank $P$ values when comparing groups. ${ }^{3}$ Significant predictors of bridge to transplantation were identified by logistic regression models. All data were analyzed with SPSS 11.5 software (SPSS, Inc, Chicago, Ill).

\section{Results}

Demographics. Clinical characteristics of patients are shown in Table 1. The number of patients in groups 1,2 , and 3 was 41 (20.4\%), $104(51.7 \%)$, and $56(27.9 \%)$, respectively. There were

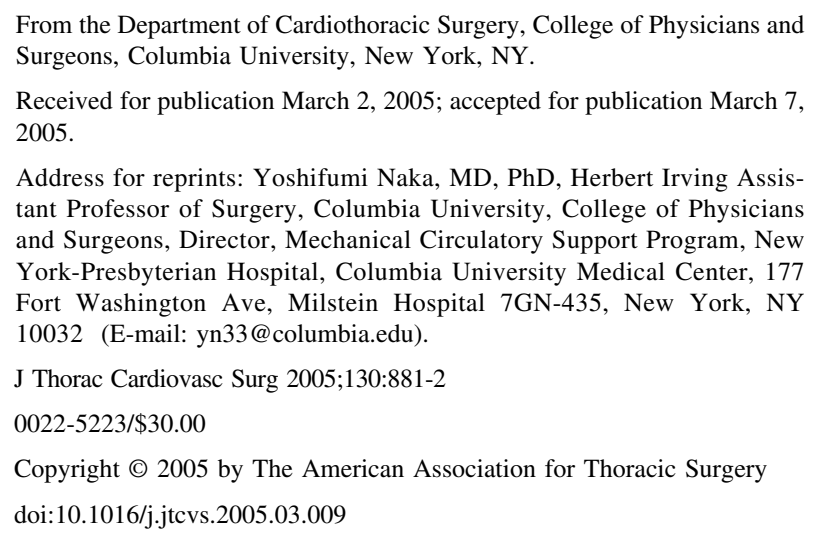

no significant differences in sex, mean body mass index, and mean LVAD score among the 3 groups. Coronary artery disease as the cause of heart failure was significantly more common in older patients, whereas idiopathic cardiomyopathy and other causes were significantly more common in younger cohorts. There was a greater incidence of hypertension in older patients undergoing LVAD implantation.

\section{Postoperative complications and bridge-to-transplanta-} tion rates. $\mathrm{LVAD}$ pocket infections and any type of infection in general were more frequent in group 2 compared with in groups 1 and 3. Postoperative stroke occurrence did not differ between groups, whereas acute renal failure necessitating continuous venovenous hemodialysis was significantly more common in group 3 than in groups 1 or 2 . Mean intensive care unit (ICU) length of stay was significantly longer in group 3 compared with the younger cohorts. Overall bridge-to-transplantation rates were comparable among the 3 age groups (Table 1).

Posttransplantation survival. Posttransplantation actuarial survivals at 1,3 , and 5 years were $93.6 \%, 86.4 \%$, and $81.1 \%$ for group $1 ; 90.1 \%, 86.7 \%$, and $78.6 \%$ for group 2 ; and $84.1 \%, 76.9 \%$, and $69.6 \%$ for group $3(P=.505, \log$-rank test; Figure 1$)$.

Predictors of bridge to transplantation. Univariate analysis revealed cause of heart failure $(P=.015$ for coronary artery disease and $P=.004$ for idiopathic cardiomyopathy), LVAD score $(P<.001)$, and female sex (trend toward significance, $P=.066)$ as predictors of bridge to transplantation. Multivariate analysis revealed only LVAD score as a significant independent predictor of bridge to transplantation (hazard ratio, $0.730 ; 95 \%$ confidence interval, 0.631-0.845; $P<.001$ ). Patients with low LVAD scores demonstrated higher bridge-to-transplantation rates compared with those with high LVAD scores. Age of 60 years or older was not a significant predictor of bridge to transplantation in either univariate or multivariate analyses.

\section{Discussion}

In our review older patients exhibited significantly higher rates of heart failure caused by coronary artery disease, as well as a higher preoperative incidence of hypertension. These findings are consistent with previous reports. ${ }^{3}$ The mean intensive care unit stay was significantly longer for elderly patients, an outcome likely related to a corresponding increased incidence of postoperative renal failure requiring continuous venovenous hemodialysis. Interestingly, advanced age had no effect on either bridge to transplantation or posttransplantation survival. This might be expected, however, because several recent reports demonstrate comparable posttransplantation survivals for older recipients as a result of improvements in surgical technique, anesthesia management, and perioperative care of patients. ${ }^{4}$ 
TABLE 1. Demographics and clinical characteristics

\begin{tabular}{|c|c|c|c|c|c|}
\hline & & Age $<40$ y & Age 40-59 y & Age $\geq 60 y$ & $P$ value \\
\hline Age (y) & & $26.6 \pm 8.8$ & $52.2 \pm 5.6$ & $63.1 \pm 2.7$ & $<.001$ \\
\hline Sex & Female & $24.4 \%$ & $17.4 \%$ & $14.3 \%$ & .428 \\
\hline \multirow[t]{3}{*}{ Race } & White & $51.2 \%$ & $71.2 \%$ & $78.6 \%$ & .011 \\
\hline & Black & $26.8 \%$ & $14.3 \%$ & $16.0 \%$ & .197 \\
\hline & Other & $22.0 \%$ & $13.5 \%$ & $5.4 \%$ & .054 \\
\hline \multirow[t]{3}{*}{ Cause of HF } & CAD & $17.1 \%$ & $60.6 \%$ & $76.8 \%$ & $<.001$ \\
\hline & ICM & $56.1 \%$ & $33.6 \%$ & $17.8 \%$ & $<.001$ \\
\hline & Other & $26.8 \%$ & $5.8 \%$ & $5.4 \%$ & $<.001$ \\
\hline LVAD score* & & $4.3 \pm 3.4$ & $4.3 \pm 3.5$ & $4.9 \pm 3.5$ & .611 \\
\hline Hypertension & & $10.0 \%$ & $33.3 \%$ & $37.3 \%$ & .009 \\
\hline BMI $\left(\mathrm{kg} / \mathrm{m}^{2}\right)$ & & $27.5 \pm 6.5$ & $27.1 \pm 5.2$ & $26.5 \pm 4.8$ & .640 \\
\hline \multirow[t]{4}{*}{ Infection } & Driveline & $5.0 \%$ & $6.1 \%$ & $4.0 \%$ & .858 \\
\hline & Pocket & $17.5 \%$ & $17.3 \%$ & $8.0 \%$ & .279 \\
\hline & Pump & $2.5 \%$ & $9.2 \%$ & $0.0 \%$ & .042 \\
\hline & Any & $35.0 \%$ & $55.1 \%$ & $37.3 \%$ & .034 \\
\hline CVVHD & & $14.6 \%$ & $35.3 \%$ & $38.2 \%$ & .028 \\
\hline Stroke & & $7.3 \%$ & $4.8 \%$ & $7.1 \%$ & .771 \\
\hline ICU LOS (d) & & $13.1 \pm 14.9$ & $14.5 \pm 11.6$ & $22.6 \pm 28.7$ & .020 \\
\hline Bridge to transplantation & & $82.5 \%$ & $73.5 \%$ & $66.7 \%$ & .228 \\
\hline
\end{tabular}

$H F$, Heart failure; $C A D$, coronary artery disease; ICM, idiopathic cardiomyopathy; $L V A D$, left ventricular assist device; $B M I$, body mass index; $C V V H D$, continuous venovenous hemodialysis; $I C U$, intensive care unit; $L O S$, length of stay. ${ }^{*} 0 f 10$ possible points.

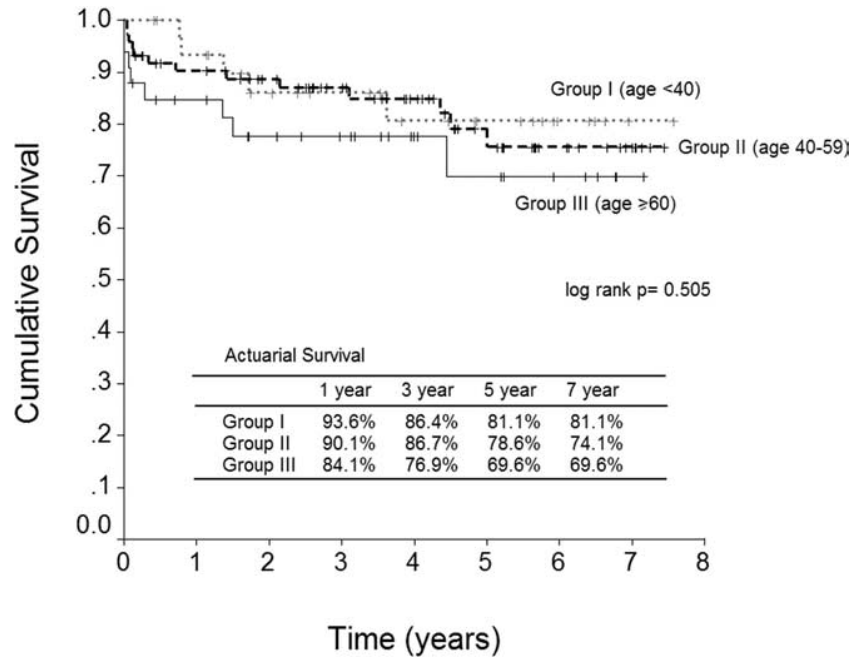

Figure 1. Posttransplantation actuarial survival.

Preimplantation LVAD score, which has previously been shown to correlate with mortality, was the only independent predictor of outcome but was otherwise comparable among the different age cohorts.
Limitations of this study include those inherent with a retrospective analysis. Data were collected retrospectively by means of chart review and might not be as accurate as data obtained in a prospective and controlled fashion.

In conclusion, LVADs can successfully bridge older patients to transplantation, with posttransplantation survivals equivalent to those achieved by younger LVAD recipients. Decisions to implant LVADs should not be based solely on age; rather, individualized preoperative patient status should be more heavily considered in the decision-making process.

\section{References}

1. Tsai TP, Chaux A, Matloff JM, Kass RM, Gray RJ, DeRobertis MA, et al. Ten-year experience of cardiac surgery in patients aged 80 years and over. Ann Thorac Surg. 1994;58:445-51.

2. Rao V, Oz MC, Flannery MA, Catanese KA, Argenziano M, Naka Y. Revised screening scale to predict survival after insertion of a left ventricular assist device. J Thorac Cardiovasc Surg. 2003;125:855-62.

3. Rich MW. Epidemiology, pathophysiology, and etiology of congestive heart failure in older adults. J Am Geriatr Soc. 1997;45:968-74.

4. Morgan JA, John R, Weinberg AD, Remoli R, Kherani AR, Vigilance $\mathrm{DW}$, et al. Long-term results of cardiac transplantation in patients 65 years of age and older: a comparative analysis. Ann Thorac Surg. 2003;76:1982-7. 